\title{
TECHNO-ECONOMIC ANALYSIS OF DISPERSION-TOLERANT TRANSMISSION TECHNIQUES FOR 10GB/S DWDM SYSTEMS
}

Cornelius Fürst, Helmut Griesser, Jörg-Peter Elbers, and Christoph Glingener Marconi, Stuttgarter Str. 139, 71522 Backnang, Germany,"

\{cornelius.fuerst | helmut.griesser | joerg-peter.elbers | christoph.glingener\}@marconi.com

\begin{abstract}
We analyse the prospects of dispersion-tolerant transmission techniques such as electronic distortion compensation and optical duobinary modulation. Our investigation of both technical and economical aspects shows that these techniques are predominantly beneficial for Metro DWDM transmission.
\end{abstract}

\section{INTRODUCTION}

In the past years, robust transmission technologies have become a hot topic for optical core and metro networks. Whilst a number of dispersion-tolerant techniques have been proposed, the technical discussion mainly focused on two approaches. At the transmit side, optical duobinary transmission has been shown to provide superior tolerance to residual chromatic dispersion, allowing dispersion uncompensated transmission over $200 \mathrm{~km}$ of standard fibre [1]. At the receive side, electrical distortion compensation (EDC) has been investigated [2], compensating for chromatic dispersion of several thousands of $\mathrm{ps} / \mathrm{nm}$ as well as several tens of $\mathrm{ps}$ of polarisation mode dispersion (PMD).

* The work reported in this paper has been supported in part by the German Ministry of Education and Research (BMBF) under contract number 01 BP 260. The authors are responsible for the content of this paper. 
Up to now there is no clear picture, under which conditions these technologies really exhibit their claimed technical benefits and, more importantly, do generate measurable cost savings in core and metro networks.

In this paper, we carry out a techno-economic analysis which indicates the network scenarios where techniques as duobinary transmission and EDC are beneficial for $10 \mathrm{~Gb} / \mathrm{s}$ DWDM systems. From the technical point of view, we investigate the dispersion tolerance provided by the these techniques for Metro and (Ultra) Long Haul systems. The economical aspect is illustrated by calculating the relative system cost savings that may be realised when a certain cost premium for each transmitter/receiver pair is assumed.

\section{DUOBINARY TRANSMISSION}

Duobinary encoding is implemented by quarter-rate filtering ( $10^{\text {th }}$ order Bessel filter) of an NRZ electrical signal. The signal then drives a Mach-Zehnder modulator at an amplitude of $2 \mathrm{Vpi}$, generating a three-level signal for the electrical field (two levels for the intensity) with narrower optical bandwidth compared to conventional NRZ coding [1,3-5].

Assuming that the width of the optical filters (e.g. multiplexers) is much larger than the signal bandwidth, duobinary transmission shows a degradation of the back-to-back performance compared to the optimum performance ( $Q$ value), obtained for residual dispersion of approx. $+/-2000 \mathrm{ps} / \mathrm{nm}$ at a bit rate of $10.7 \mathrm{~Gb} / \mathrm{s}$ (see inset of Figure 1). Approximately $1.5 \mathrm{~dB}$ of OSNR margin has to be allowed to obtain a dispersion tolerance of $+3000 \mathrm{ps} / \mathrm{nm}$ (180 km of standard fibre). Spending another $2 \mathrm{~dB}$ extends the tolerance to $+3500 \mathrm{ps} / \mathrm{nm}$.

That behaviour changes fundamentally after transmission of the signal over long-haul and ultra-long haul distances. Figure 1 shows simulation results of a single channel propagated over 25 spans of each $80 \mathrm{~km}$ standard fibre. Doublestage amplifiers compensate the fibre loss $(20 \mathrm{~dB})$ and contain a dispersion compensating fibre (DCF) as an interstage device. Fibre input power levels of $-3 \mathrm{dBm}$ to $+5 \mathrm{dBm}$ into the standard fibre and $-2 \mathrm{dBm}$ into the DCFs are chosen. The performance does not show anymore the "M"-shape. The dispersion tolerance is substantially reduced, even for moderate power levels. Figure $I b$ compares the results for duobinary and NRZ showing that the behaviour of both formats practically converges under the influence of fibre nonlinearities.

We have analysed the dispersion tolerance under various conditions, especially when varying the dispersion map (precompensation and slope of the accumulated dispersion). Figure 2 shows the $-1 \mathrm{~dB}$ width of the Q-vs-dispersion curves for 25 spans of DWDM transmission at $50 \mathrm{GHz}$ channel spacing. 

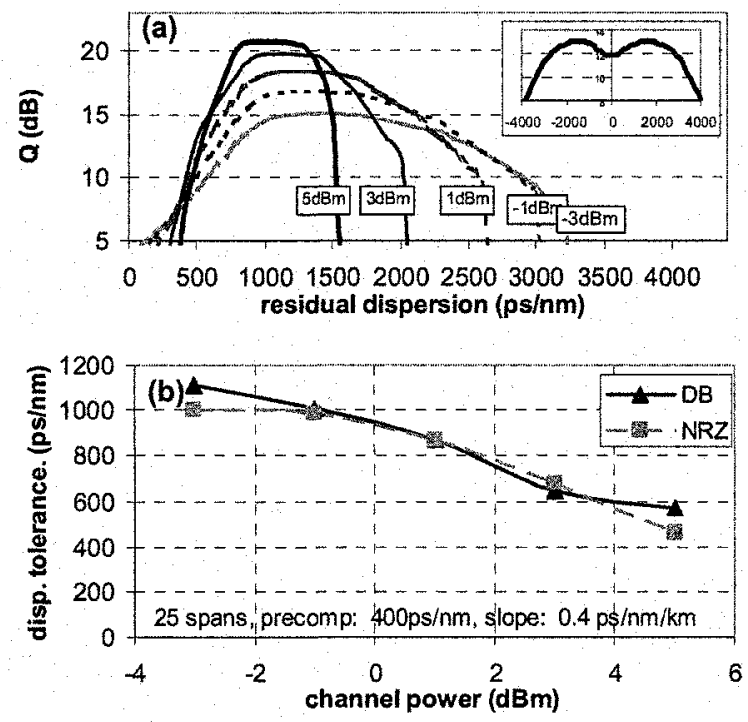

Figure 1. Dispersion tolerance of optical duobinary signals after transmission over 25 spans: (a) Simulated $Q$ for different channel launch power levels into the standard fibre spans. Inset: Linear transmission showing the ' $\mathrm{M}$ '-shape, symmetric to zero dispersion.

(b) Simulated nonlinear dispersion tolerance compared to NRZ.

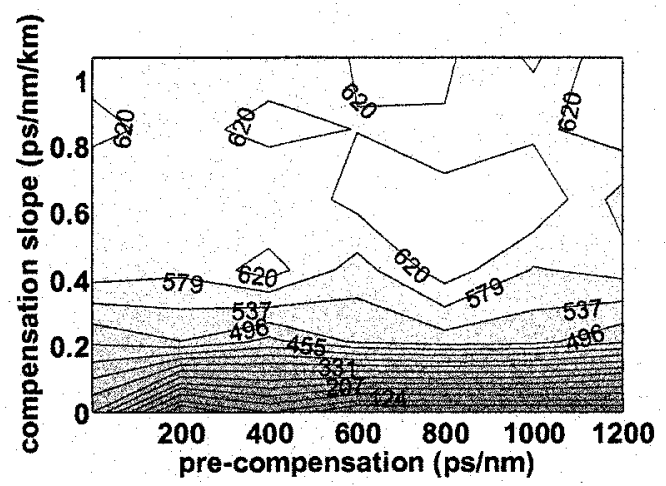

Figure 2. Behaviour of the dispersion tolerance (-1dB width of the Q-vs-dispersion curves) in $\mathrm{ps} / \mathrm{nm}$ in dependence of the dispersion map parameters.

For a slope of the accumulated dispersion of $>0.3 \mathrm{ps} / \mathrm{nm} / \mathrm{km}$, a low dispersion tolerance of $600 \mathrm{ps} / \mathrm{nm}$ is found, similar to the behaviour of NRZ. Low slope of the accumulated dispersion $(<0.2 \mathrm{ps} / \mathrm{nm} / \mathrm{km}$ ) has to be avoided to prevent resonant 
accumulation of distortion due to cross-phase modulation (XPM). The only improvement found with respect to NRZ is a slightly better tolerance of variations of the dispersion map (e.g. practically no influence of the precompensation).

We can conclude that whilst optical duobinary modulation is facilitator for dispersion-compensating fibre (DCF) free transmission in the Metro range (up to $200 \mathrm{~km}$ ) there is very limited technical benefit for LH and ULH scenarios.

\section{ELECTRONIC DISTORTION EQUALISATION}

Our assessment of electronic distortion equalisers is concentrated on maximum likelihood sequence estimation (MLSE) [6] due to its powerful distortion mitigation capabilities, but, for comparison, also includes simpler equalisers schemes such as feed-forward (FFE) and decision-feedback equalisers (DFE).

The optically pre-amplified receiver front-end consists of an EDFA and a $50 \mathrm{GHz}$ super-Gaussian filter followed by a photodiode and a $7.5 \mathrm{GHz} 5^{\text {th }}$ order Bessel filter. Only optical amplifier noise is considered.

The simulation model for the MLSE is based on a moderate 4 state trellis (with corresponds to a channel memory of 2) and a 4 bit signal quantisation with twofold oversampling [7]. The simulation for the FFE assumes 6 taps, fractionally spaced by $\mathrm{T} / 2$ (where $\mathrm{T}$ is the bit time), and for the DFE 4 feed-forward and 2 feedback taps, also with a spacing of $\mathrm{T} / 2$, are considered.

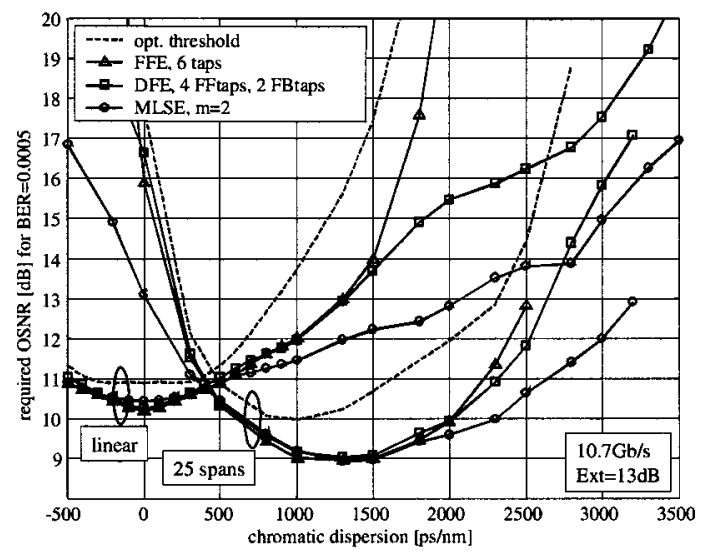

Figure 3. Dispersion tolerance of EDC receivers after a linear single span and a 25 span transmission of a single $10.7 \mathrm{~Gb} / \mathrm{s}$ channel. 
The system performance is evaluated by means of Monte-Carlo simulations. It is expressed as required optical signal to noise ratio (OSNR) in $0.1 \mathrm{~nm}$ bandwidth) to reach a bit error rate (BER) of 5e-4, sufficient for error-free operation with enhanced FEC.

Similar to the previous chapter, we compare the dispersion tolerance for the linear case (distance $<300 \mathrm{~km}$, low channel power levels) and for transmission after 25 spans of standard fibre with a launch power of $1 \mathrm{dBm}$. In the linear case, we find a dispersion tolerance of $3000 \mathrm{ps} / \mathrm{nm}$ when spending an OSNR margin of $\sim 4 \mathrm{~dB}$ (Figure 3). The figure also presents results for the ULH transmission, with MLSE providing a clear improvement with respect to the conventional optimum threshold receiver. Also the FFE and DFE equalisers enhance the dispersion tolerance, with some reduced gain for higher values of the residual dispersion. From these results, we can conclude that EDC technologies keep their technical benefit for a wide range of network applications.

\section{ECONOMICAL ANALYSIS}

In this section, we provide an general investigation of the cost benefits that can be realised by the implementation of dispersion tolerant transmission techniques. The analysis treats the dispersion tolerance, the required OSNR margin and the cost premium (with respect to standard NRZ transmitters and receivers) as parameters, therefore providing results which are independent of the technique actually employed.

We consider cost changes related to the reduction of the quantity of equipment needed to set up a system and take into account the change of the costs of components by using the alternative technologies (e.g. EDC). Cost savings related to operational benefits are not considered as they are typically difficult to quantify.

Two network scenarios are chosen for the analysis: (1) A DWDM Metro ring network, comprising distances between $100 \mathrm{~km}$ and $250 \mathrm{~km}$ and a number of nodes between 5 and 8 . Each network node contains a fixed OADM and, depending on the loss between the nodes, one amplifier pair (bidirectional). (2) A DWDM Long Haul Network at distances between 500 and $1500 \mathrm{~km}$. We use a simplified cost basis used for the calculations, reflecting a typical relationship of the costs of the main system components. For the costs of a $2.5 \mathrm{G}$ transponder normalised to 1 , we assume the $10 \mathrm{G}$ transponder cost as 2.5 , a single stage amplifier as 1.25 and a double stage amplifier (providing interstage access for DCMs) as 2. The DCM costs scale with the length $L$ of compensated standard fibre as $0.25+0.01 \times \mathrm{L}$. 


\subsection{Metro scenario}

Most traffic today is a maximum $2.5 \mathrm{G}$ traffic intrinsic bandwidth. The introduction of $10 \mathrm{G}$ traffic needs handling of the chromatic dispersion, predominantly in standard G.652 networks. In order to allow in-service upgrade, dispersion compensation needs to be implemented during the installation of the system and therefore heavily affects the first-in costs of the system. Especially here, tolerant transmission techniques help to achieve acceptable economics for 10G upgrade traffic by saving capital expenditures (CAPEX). Actual cost savings are expressed by two items: (1) Partial or full replacement of DCM modules. (2) Potential saving of a single EDFA due to reduced network loss when DCMs are removed.

Figure 4 presents savings of first-in costs including $8 \times 2.5 \mathrm{G}$ traffic. Rather than concentrating on an arbitrary length of the network, the results are averaged over system lengths from 100 to $250 \mathrm{~km}$. Cost savings of $15 \%$ are realised for an average network.
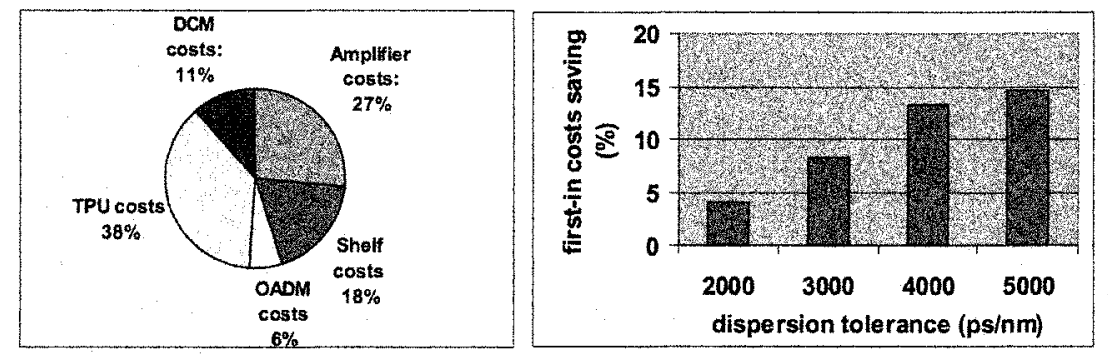

Figure 4. Assumed cost contribution and saving of first-in costs in a 8x2.5G Metro network.

Figure 5 presents the cost saving relative to the total system costs after upgrading to $8 \times 10 \mathrm{G}$ vs. the $10 \mathrm{G}$ transponder cost premium and the achieved dispersion tolerance. For a dispersion tolerance of $3000 \mathrm{ps} / \mathrm{nm}$, we find the break even point at a cost premium of $8 \%$ of the $10 \mathrm{G}$ transponders. The same calculation for 24 channels at $10 \mathrm{G}$ shows a break even point at $4 \%$.

If the technology needs some extra OSNR margin (e.g. EDC needs $4 \mathrm{~dB}$ for $3000 \mathrm{ps} / \mathrm{nm}$, duobinary $2 \mathrm{~dB}$ for $3000 \mathrm{ps} / \mathrm{nm}$ ), cost savings are reduced since the saving of an amplifier becomes improbable. From further calculations we see that that the averaged savings are $0.5 \%$ lower than in the previous case at low OSNR penalty. Note that the obtained figures are averaged over a range of network extensions, and therefore individual first-in cost savings may be considerably higher. 


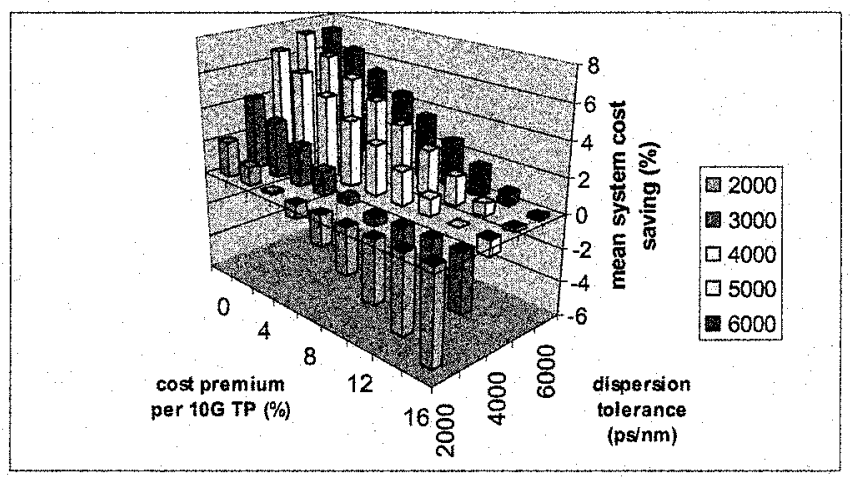

Figure 5. Savings in a Metro network loaded by $8 \times 2.5 \mathrm{G}$ and $8 \times 10 \mathrm{G}$ traffic.

\subsection{Long-Haul scenario}

In the LH case, cost savings are expected to be much lower since the actual technologies can compensate for only a small part of the dispersion of these networks. Savings are realised by (1) simplifying the dispersion map which allows to replace double-stage amplifiers by cheaper single stage ones and (2) removing the terminal DCFs at the receive side. Figure 6 shows for a 8-channnel system that savings are in the range of few percent and are rapidly outweighed by the transponder cost premium for rising channel count. For a fully utilised system with 40 channels, cost savings are only realised when the cost premium for a $3000 \mathrm{ps} / \mathrm{nm}$ transponder technology is below 3\%, imposing considerable cost pressure onto any technique for enhanced chromatic dispersion tolerance.

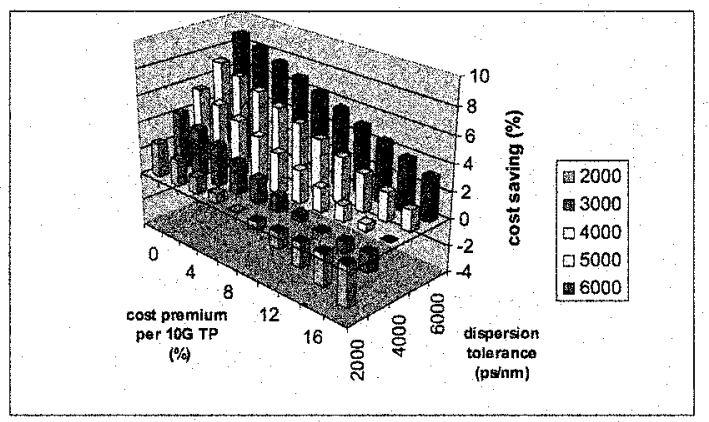

Figure 6. Cost savings relative to the total system costs for $\mathrm{LH}$ networks at distances between 500 and $1200 \mathrm{~km}$ and a load of $8 \times 10 \mathrm{~Gb} / \mathrm{s}$. 


\section{CONCLUSIONS}

Duobinary modulation and electronic distortion compensation are promising technologies to realise cost savings in future DWDM Metro networks at 10Gb/s line rates. Both technologies provide a dispersion tolerance that enables the seamless upgrade of $2.5 \mathrm{~Gb} / \mathrm{s}$ systems to $10 \mathrm{~Gb} / \mathrm{s}$ for $<200 \mathrm{~km}$ links, without provoking excessive first-in costs. By employing these techniques, first-in cost savings around $15 \%$ can be realised compared to systems with conventional dispersion compensation. In order to prevent cost penalties at higher channel population, the cost premium should be below about $4 \%$ of the original transponder costs when the technology is capable of tolerating $3000 \mathrm{ps} / \mathrm{nm}$ of dispersion.

For Long-haul systems, the situation is different. Due to impact of fibre nonlinearities, duobinary transmission does not provide superior dispersion tolerance anymore. EDC still increases the dispersion tolerance for the long-haul case, albeit the benefit is reduced compared to the linear transmission. Potential cost savings for any dispersion tolerant technology are clearly lower than in a Metro network and the acceptable transponder cost premium to avoid cost penalties at higher channel counts is even lower.

The cost estimations do not include some potential savings, which are difficult to take into account. There may be operational benefits such as a simpler installation procedure and design benefits such as relaxed specifications for DCMs in ULH networks. Additionally, dispersion-tolerant technology can simplify the setup of optical transparent networks, where the dispersion management is more difficult due to the diversity of optical paths.

\section{REFERENCES}

[1] S. Walklin et al., IEEE Photon. Technol. Lett., Vol. 9 (1997), page 1005.

[2] Haunstein et al., OFC 2001, paper WAA4-1.

[3] D. Penninckx et al., IEEE Photon. Technol. Lett., Vol. 9 (1997), page 259.

[4] H. Bissessur et al., OFC 2001, paper WDD36.

[5] W. Kaiser et al., ECOC 2000, paper 7.2.2.

[6] Benedetto et al., Digital Transmission Theory, Prentice Hall, 1987.

[7] Fludger et al., OFC 2004, paper WM7. 